\title{
OUTPUT CURRENT CONTROL SYSTEM OF A HIGH VOLTAGE ELECTRIC PULSE GENERATOR FOR PLASMA EXCITATION
}

\author{
D.V. Godun*, S.V. Bordusau, G.P. Budzko \\ Belarusian State University of Informatics and Radioelectronics, P. Brovki 6, 220013, Minsk, Belarus \\ * godundv@mail.ru
}

\begin{abstract}
A control and pulse discharge current limiting system integrated into an AC/DC converter and pulse modulator of a high voltage pulse generator have been developed. The peculiarity of such system's operation is the stabilization of the power supplied to the discharge and the correction of the width of output electric pulses towards decrease upon reaching the specified pulsed current amplitude value. The system enables the pulse generator to work in the modes close to the "short circuited load" mode. In this case the driving module of a composite IGBT key performs the correction of the working pulse width and blocks the pulse generator operation if needed. The suggested circuit design solutions allow using the generator in a wide range of electric plasma-forming parameters' modes and working with various types of vacuum gas discharge systems.
\end{abstract}

Keywords: output current, control, electric pulses, plasma load.

\section{Introduction}

It is known that for working with plasma load in order to protect a generator of electric pulses from the avalanche current flowing through the open crossover of the power IGBT key, it is necessary to provide high speed electronic protection system $[1,2]$. It is due to the fact that unstable plasma load may lead to the change of its electric characteristics (as well as because of spontaneous transition into another kind of gas discharge). The speed and the value of the growing current, flowing across the power key, are directly determined by the conductivity and volume of the formed discharge zone. The stability of output electric pulse generator characteristics can be achieved with the specific circuit solutions and a modern component base.

The most important factor influencing the effective operation of a power pulse generator is the right choice of the key IGBT transistor. The key block must have the necessary frequency properties enabling it to perform commutation of the load at the specified frequencies, provided the rate of power pulses rise corresponds to the required. Non-compliance of the frequency properties of the selected key can cause a breakdown of a transistor, distortion of the power pulse fronts, the emergence of inflowing currents and the increase of the formed plasma discharge reactivity [3].

The most perspective concept of designing a power key for a universal pulse plasma discharge systems is the use of high speed discrete keys with sequential switching [4]. Such circuit solution allows to get the required steepness of the formed pulses fronts at frequencies up to $60 \mathrm{kHz}$ while switching pulses currents up to $80 \mathrm{~A}$.

Because of the peculiarities of a pulse generator application for synthesizing an unstable plasma, it is desirable to embed a system of pulse current control into an output generator circuit. Such system should provide the required time for safety shutdown and provide the function of a controlled turn off threshold. To reduce the number of false protection block's triggering, the preliminary amplifier should be based on a separate low noise operational amplifier and provide the selection of the desired signal with the help of passive filtering elements.

\section{Main part}

In reference source [5] some circuit solutions of designing a powerful high voltage electric pulse generator with the functions of stabilizing the current discharge as well as the voltage at a discharge gap are presented.

In the designed high voltage pulse generator, to provide a breakdown of gas discharge medium at different work pressure, control and a mode of stabilizing the voltage amplitude applied to the gas discharge gap are provided. The availability of such option in the pulse generator allows to control the amplitude of pulses in the interval from -600 to $-1200 \mathrm{~V}$ with the function of limiting the current in the load circuit. It allows to abandon the additional high voltage igniting devices. It makes the cost of a generator significantly lower, simplifies the construction and improves its operation reliability. The availability of an output pulse voltage control makes it possible to use the pulse generator for different discharge systems.

The discharge current stabilization mode is required to move smoothly through the voltage-current characteristic of the pulse discharge. It is also necessary to take care of limiting the pulse power consumed by the non-stationary plasma discharge. After the gas breakdown the pulse generator allows to work in different stationary modes, even close to the mode 


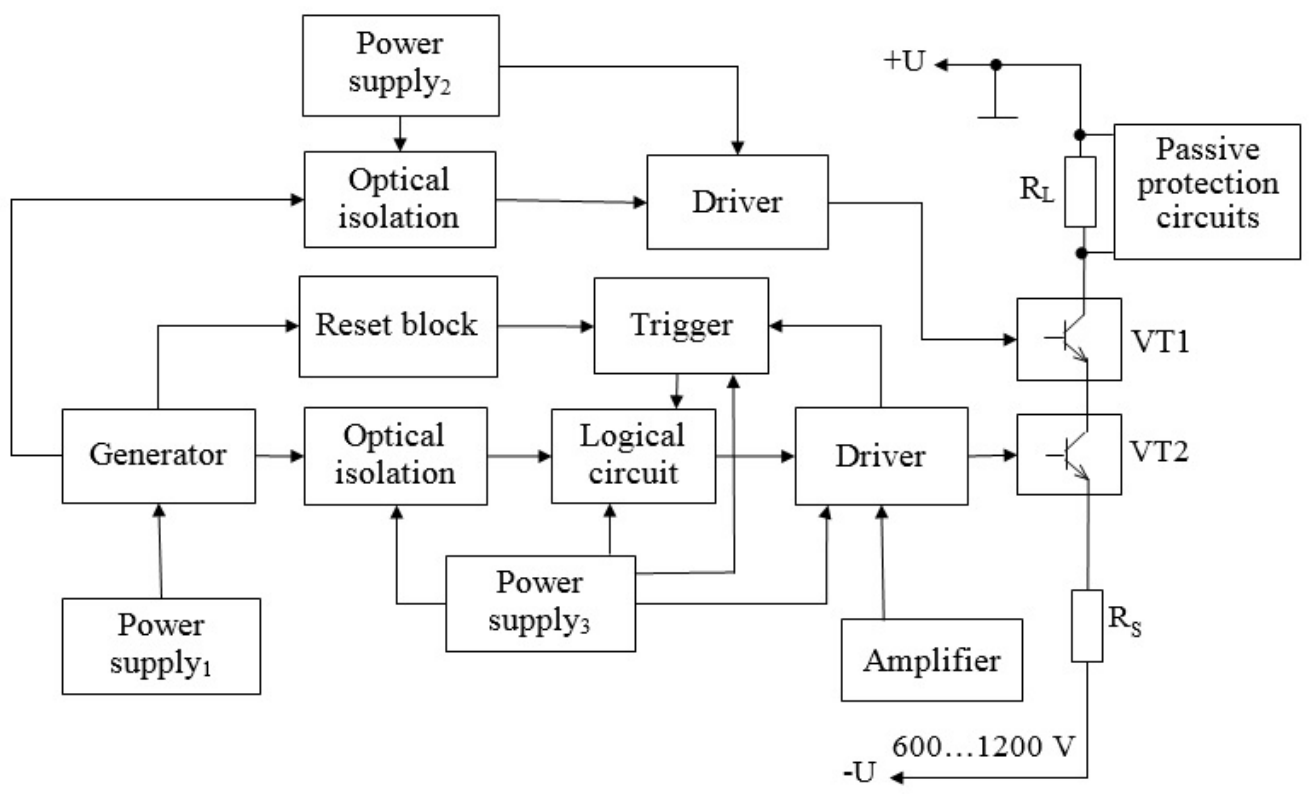

Figure 1. Structural diagram of a driver device with the function of pulse discharge current limiting

of forming an arc discharge. The implemented circuit design solutions provide a decreased probability of damaging the processed samples due to the formation of a great number of micro-arcs in a limited volume [6]. The mentioned effect can be eliminated by increasing the off-duty factor of power pulses both manually and automatically. Note, the system, in this case, increases the amplitude value of current pulses and this, in practice, ensures maintaining constant level of the pulse power applied to the discharge. The implementation of the stabilization mode of the discharge pulse current allows a significant reduction in the pulse current growth rate through the power key when operating in non-stationary modes. This is one of the most important advantages of the system under study.

An additional barrier of protecting power semiconductor devices from admissible pulse current excess is an integrated protection system built in a driver module.

A structural diagram of the designed driver module with the function of pulse current limiting is shown in Fig.1.

As it has been mentioned above, a composite pulse key on the discrete IGBT transistors connected in series is the most preferable variant of designing a pulse modulator. Because of a standard construction of the pulse modulator to control the upper key at VTI transistor, let's analyze the functioning of the lower key based on the VT2 transistor with its functional peculiarities.

The generator forms one channel of programcontrolled pulses that get simultaneously through the optical converters block to the driver module of the upper key and through the logical circuit to the driver module of the lower key. The driver forms the signals controlling the VT1 transistor. In a normal mode of system operation, the two keys must open in-phase. The logical circuit is implemented for converting signals into the required type according to the developed operation algorithm. After processing in the block of logical conversion, the pulses get to the input of the lower key driver module. The lower IGBT transistor is controlled in the same way as the upper key. In the presented structural diagram, the formed plasma nonstationary discharge is indicated as $R_{L}$. The voltage controlled AC/DC converter with the function of stabilizing the output power through $R_{L}$, the composite key and the current sensor is connected to the ground. The signal, proportional to the value of the flowing current, is taken from the sensor $R_{S}$ and is passed to the low noise amplifier with a controlled amplification coefficient. The use of passive correction circuits in the scheme of a low-noise amplifier of provides the filtration and conversion of the signal which subsequently is passed to the input of the current control of lower key driver module. The driver's micro circuit at this input differentiates two modes - overload and short circuit mode. All pulse current control systems are included into driver's microcircuit. According to the technical characteristics of the driver's microcircuit the time of the safety shutdown does not exceed 3 microseconds. This has been proved by practical experiments. When in an overload mode, the driver makes the lower key control pulse narrower, thus, limiting the average value of the pulse current flowing through the composite transistor. In the short circuit mode, the driver module blocks the operation of the lower IGBT transistor, forms an error signal which is passed to the trigger circuit and, further, a blocking signal of module operation is formed. The withdrawal of the system from this state is possible on an user's external signal, using the reset block included in the system. 

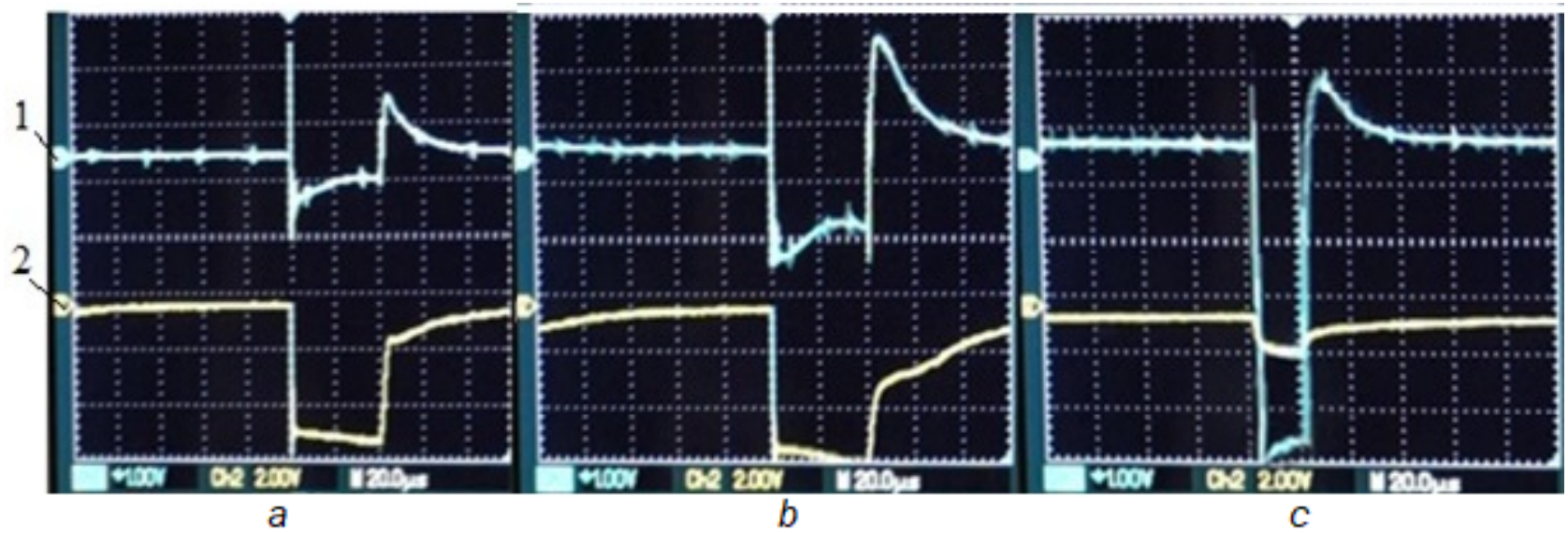

Figure 2. Oscillograms of current pulses and voltage at plasma load at $f=5 \mathrm{kHz}$; 1 - signal from current pulse; 2- pulse voltage signal

The presented device has been implemented and tested as part of the high voltage pulse generator. The examples of the developed generator's output parameters control system while working with a nonstationary plasma load for the output pulses' frequency $\mathrm{f}=5 \mathrm{kHz}$ are given in Fig. 2 .

Fig.2a shows the pulse current and voltage oscillograms at plasma load. Fig.2b shows the change of current and voltage pulses when the consumed power of the discharge increases. Fig.2c shows the oscillogaram of current and voltage pulses when the pulse power supply source works in the mode of discharge current stabilization close to the short circuit mode. One can see that when the generator works for plasma load using the function of stabilization of the power applied to the discharge, it is possible to maintain different current and voltage modes in the load, i.e. to move along the voltage-current characteristic of the discharge.

\section{Conclusion}

A system for the output current control of a highvoltage electric pulse generator has been developed and tested with the formation of a non-stationary plasma discharge. It has been experimentally established that the reaction time of the system to the excess of pulse value of the current flowing through the non-stationary discharge does not exceed 3 microseconds. Taking into account that the system already includes the circuit of current negative feedback which provides the stabilization of $\mathrm{AC} / \mathrm{DC}$ converter's output power, the rate of current growth in the load circuit in emergency situations significantly decreases and the reaction time of the system is sufficient to protect the key IGBT transistors from overloading.

\section{Acknowledgements}

This research has been supported by Belarusian State Program of Scientific Research "Science of physical materials, new materials and technologies", assignment 3.5.02

\section{References}

[1] C. Yong-Nong and K. Chih-Ming. Design of plasma generator driven by high-frequency high-voltage power supply. Journal of Applied Research and Technology, 11(2):225-234, 2013.

doi:10.4028/www.scientific.net/amm.284-287.1136. doi : 10.4028/www . scientific .net/AMM . 284-287 . 1136.

[2] J. E. Harry. Introduction to Plasma Technology: Science, Engineering, and Applications. Wiley-VCH, 2010.

[3] A. Polishchuk. Selection of key transistors for hardswitching converters. Power Electronics, (2):22-25, 2004.

[4] A. Wintrich, U. Nicolai, W. Tursky, and R. T. Problems of parallel and serial igbt connection. part 2. sequential work of the igbt. Power Electronics, (5):34-40, 2013.

[5] D. Godun, S. Bordusov, and A. Dostanko. The software-controlled generator of high-voltage impulses for plasma technological application. Acta Technica, (56):332-337, 2011.

[6] D. Godun, S. Bordusov, and A. Dostanko. Systems of electronic overcurrent protection in pulse power generator operating on plasma load. Acta Polytechnica, 53(2):152-154, 2013. 